\title{
Eutopic and Ectopic Parathyroids in Hyperparathyroidism: Incidence, Localization Trends and Diagnostic Evaluation- Retrospective Data of a Cohort of 400 Patients from a Single Tertiary Institute
}

\author{
Alka A Singhal ${ }^{1}$, Deepak Sarin ${ }^{2}$, Ambrish Mithal ${ }^{3}$, Sanjay S Baijal ${ }^{4}$
}

\begin{abstract}
Aim and objective: To evaluate the incidence of various locations of enlarged eutopic and ectopic parathyroids in patients of hyperparathyroidism, their imaging features, diagnosis, and surgical confirmation.

Materials and methods: A retrospective study conducted at a tertiary institution, where 400 patients operated for hyperparathyroidism from 2012 to 2017 was analyzed. Diagnostic findings of Tc-99m-sestamibi scan and ultrasound were compared with surgical findings and subsequent histopathology. Contrast CT(4D CT) was utilized only in select cases.

Results: Of 400 patients single adenoma was seen in 350 patients (87.5\%), two or more nodules in 41 patients (10.2\%), and carcinoma in 3 cases. Of the 350 solitary adenomas, 291 were juxtaposed to thyroid (83.1\%), and ectopic in $59(16.8 \%)$ cases. Ectopic along the thymic tract in 38 cases (10.8\%), upper mediastinum 8 cases (2.2\%), angle of jaw in 2 cases $(0.5 \%)$, carotid sheath 2 cases $(0.5 \%)$,retroesophageal 2 cases $(0.5 \%)$ and intrathyroidal in 7 cases (2\%). Tc-99m-sestamibi scan was positive in localizing parathyroid nodules in 297 (84.8\%) cases, and ultrasound in $339(96.8 \%)$ cases. Of the nodules localized by Tc- $99 \mathrm{~m}$-sestamibi scan, $82 \%$ were true and $3 \%$ were false localization at the surgery. Nodules localized by ultrasound were true in $99 \%$ and false in $1 \%$ cases. Ultrasound localized the nodules in sestamibi negative cases in $12 \%$ of patients. Conclusion: Identification of enlarged parathyroids and their differentiation from thyroid nodules and other neck lesions is the key role of imaging. Ultrasound is complimentary to Tc- $99 \mathrm{~m}$-sestamibi scan and offers significant value addition by precision anatomical localization, characterization and finding additional nodules. Using two imaging modalities of Tc-99m-sestamibi scan and ultrasound, the diagnostic sensitivity is $99 \%$ and specificity is $98 \%$.

Clinical significance: Localization of all the abnormal parathyroid glands in hyperparathyroidism remains a diagnostic challenge on account of both sensitivity and specificity of the available imaging modalities, as well as anatomical variations in the location of the parathyroid glands. A thorough knowledge of the anatomy, embryology along with the merits and pitfalls of the various available imaging modalities is essential to make an accurate preoperative diagnosis and avoid revision surgeries.

Keypoints:

- Parathyroid glands are ectopic in $16.8 \%$ cases.

- Technetium Tc-99m-sestamibi localizes the parathyroid nodules in $85 \%$ cases.

- Ultrasound detects parathyroid nodules in additional sestamibi negative cases (12\%).

- Ultrasound gives precise anatomical localization of parathyroid nodules.

- With both MIBI and ultrasound, the diagnostic sensitivity is $99 \%$ and specificity is $98 \%$.

Keywords: Cohort study, Ectopic parathyroid, Eutopic parathyroid, Hyperparathyroidism, Parathyroid adenoma, Parathyroid carcinoma, Tc-99msestamibi scan, Technetium-99m, Ultrasound imaging.

World Journal of Endocrine Surgery (2020): 10.5005/jp-journals-10002-1291
\end{abstract}

\section{INTRODUCTION}

Localization of the abnormal parathyroid glands in cases of hyperparathyroidism remains a diagnostic challenge both for the nuclear physician and for the radiologist. Various diagnostic modalities are utilized. ${ }^{1}$ The usual first-line investigation at our institute is Tc-99m-sestamibi scan, followed by ultrasound. Another cross-sectional imaging, dynamic 4D CT scan was utilized in select cases. The diagnostic dilemma arises both from the anatomical variability in the location of the parathyroids, ${ }^{2}$ and the sensitivity of the various available diagnostic modalities. Role of preoperative imaging is very vital in localization of all the abnormal parathyroid glands for effective surgical management.
${ }^{1,4}$ Division of Radiology and Nuclear Medicine, Medanta: The Medicity, Gurugram, Haryana, India

${ }^{2}$ Department of Head and Neck Oncosurgery, Medanta: The Medicity, Gurugram, Haryana, India

${ }^{3}$ Division of Endocrinology and Diabetes, Medanta: The Medicity, Gurugram, Haryana, India

Corresponding Author: Alka A Singhal, Division of Radiology and Nuclear Medicine, Medanta: The Medicity, Gurugram, Haryana, India, Phone: +91 9958938484, e-mail: singhaldralka@gmail.com

How to cite this article: Singhal AA, Sarin D, Mithal A, et al. Eutopic and Ectopic Parathyroids in Hyperparathyroidism: Incidence, Localization Trends and Diagnostic Evaluation-Retrospective Data of a Cohort

(c) The Author(s). 2020 Open Access This article is distributed under the terms of the Creative Commons Attribution 4.0 International License (https://creativecommons. org/licenses/by-nc/4.0/), which permits unrestricted use, distribution, and non-commercial reproduction in any medium, provided you give appropriate credit to the original author(s) and the source, provide a link to the Creative Commons license, and indicate if changes were made. The Creative Commons Public Domain Dedication waiver (http://creativecommons.org/publicdomain/zero/1.0/) applies to the data made available in this article, unless otherwise stated. 
It allows the surgeon to do a focussed surgery and avoids bilateral neck exploration (BNE). It reduces failed surgeries and revision surgeries.

\section{Anatomy and Embryology of Parathyroid GLANDS}

\section{Anatomy and Typical Locations}

The parathyroid glands are typically four in number, paired superior and inferior parathyroid glands, although supernumerary parathyroids ${ }^{3}$ have also been described with a reported incidence of up to $13 \%$.

Normal parathyroid glands are small and typically measure between $6 \mathrm{~mm}$ in length and 3-4 $\mathrm{mm}$ in transverse dimensions. ${ }^{4}$

The superior parathyroid glands are typically located posterior to the mid part of the thyroid lobe in about $85 \%$ cases. The inferior parathyroid glands are typically located within a $1 \mathrm{~cm}$ radius along the lower pole of thyroid in majority cases, either inferior, lateral, or posterior to the lower pole or just within the lower capsule of the thyroid gland.

\section{Embryology and Ectopic Locations}

The parathyroid glands develop from the endoderm of the third and fourth pharyngeal pouches beginning at the fifth week of embryonic life. ${ }^{5,6}$

\section{Superior Parathyroid Glands}

The superior parathyroid glands arise from the endoderm of the fourth pharyngeal pouch. During the sixth week of embryonic development, they descend along with the thyroid, usually in close relation to the posterior aspect of mid thyroid lobe. The typical location of superior parathyroids is just above or within a centimeter radius of the crossing of the inferior thyroid artery and the recurrent laryngeal nerve on the posterior mid surface of the thyroid. ${ }^{2,7}$

As the superior parathyroids have a smaller descent as compared to inferior parathyroids, they are less likely to show anatomical variability and ectopic locations. The commonest ectopic location of superior parathyroids is behind the upper pole of the thyroid, seen in about $2 \%$ cases and rarely beyond and superior to the upper pole in less than $1 \%$ cases. Ectopic superior parathyroids may be found in parapharyngeal or paraesophageal, retropharyngeal or retroesophageal locations, or maybe pushed down the tracheoesophageal groove. They may be intrathyroidal in location.

\section{Inferior Parathyroid Glands}

The inferior parathyroid glands arise from the endoderm of the third pharyngeal pouch along with the thymus and descent along with them infero-medially towards the upper mediastinum. The inferior parathyroids separate from the thymus before it enters into the mediastinum, and usually localize close to the lower pole of the thyroids. However, any aberration to their separation and descent accounts for the significant anatomical variability in their localization. They could be undescended, overdescended or trapped within the thyroid gland. Ectopic inferior parathyroids may be undescended, located as high up as the angle of the jaw, in the carotid bulb, or overdescended into the superior mediastinum. They may be intrathymic or intrathyroidal or be found anywhere along the carotid sheath. An expert and a thorough knowledge of the possible ectopic locations is the key first step in the diagnostic evaluation of the parathyroid glands. of 400 Patients from a Single Tertiary Institute. World J Endoc Surg 2020;12(2):66-75.

Source of support: Nil

Conflict of interest: None

The parathyroid glands show contralateral symmetry of location, with superior parathyroid glands in $80 \%$ cases, and inferior parathyroids in only $70 \%$ cases.

The superior and inferior parathyroid gland nomenclature is based on the embryologic origin of the parathyroid and not by their level of location in the neck. The inferior parathyroid gland may be located at the same or higher level than the superior parathyroid (in case of undescended ectopic inferior parathyroids).

Supernumerary parathyroids are very rare, ${ }^{8-11}$ and have been reported in some series to be up to $12.2 \%$ in cases of multiple endocrine neoplasia type 1 (MEN1) syndromes. They may lead to failed surgery or persistent hyperparathyroidism (HPT).

\section{Materials and Methods}

This is a retrospective study performed at a single tertiary institute. Patients operated for hyperparathyroidism from 2012 to 2017 at the institute were analyzed. The sample size was of 400 patients. Diagnostic findings of sestamibi and ultrasound were compared with surgical findings. Single-photon emission computerized tomography (SPECT) was used in select cases.

\section{Results}

Of the 400 patients, parathyroid nodules were found in perithyroid locations in $84 \%$ cases, and ectopic locations in $16 \%$ cases. For the juxtathyroid parathyroids, the sensitivity of localization by ultrasound was $98 \%$ and with MIBI Scan was $78 \%$. For the ectopic parathyroids in the neck, the sensitivity of localization by ultrasound was $95 \%$ and with sestamibi was $69 \%$. Ultrasound detected additional nodules over those detected by MIBI in $16 \%$ cases. Ultrasound localized parathyroid nodules in sestamibi negative HPT in $12 \%$ cases. Commonest perithyroid location was right inferior parathyroid. Ectopic locations were as follows; thyrothymic tract $10.8 \%$, angle of jaw $0.5 \%$, tracheoesophageal and periesophageal $0.5 \%$, carotid sheath $0.5 \%$, and intrathyroidal parathyroids $2 \%$. Mediastinal ectopic parathyroid was $1.7 \%$. On histopathology $85.4 \%$ were parathyroid adenoma, $11 \%$ were parathyroid hyperplasia, and $3.6 \%$ were parathyroid carcinoma.

\section{Hyperparathyroidism: Bioclinical Features}

An understanding of the serum calcium and parathyroid hormone levels is fundamental to the diagnostic evaluation. Normal serum calcium levels for adults are between $8.4 \mathrm{mg} / \mathrm{dL}$ and $10.2 \mathrm{mg} / \mathrm{dL}$. The normal range for adult parathyroid hormone (serum iPTH) levels is $15-68 \mathrm{pg} / \mathrm{mL} .{ }^{11}$ Levels higher than these values suggest hyperparathyroidism. Correlation with other bioclinical parameters of vitamin $D$, phosphates and others is done to establish the diagnosis. ${ }^{12}$ Mean serum calcium levels noted in this study was 11.9 $\mathrm{mg} / \mathrm{dL}$. Mean elevated PTH levels were $360 \mathrm{pg} / \mathrm{mL}$. Very high serum calcium levels (>14 $\mathrm{mg} / \mathrm{dL}$ ) and very high parathyroid hormone levels, in thousands $(>4,500 \mathrm{pg} / \mathrm{dL}$ ) are suspicious of parathyroid carcinoma. ${ }^{13,14}$ Most patients in this study had mild symptoms of weakness or bone/muscle pain. Common associations with renal calculi or pancreatitis were noted. 


\section{Tc-99m-sestamibi Scan for Parathyroids}

Tc-99m-sestamibi is commonly used to localize parathyroid adenoma either as combined subtraction procedure with $I^{123}$ or $\mathrm{TcO}_{4}$ or indeed as a sole agent. The radiotracer localizes and is retained in the region of mitochondria. Parathyroid adenoma's high cellularity and vascularity and large number of mitochondria in oxyphil cells are responsible for its avid uptake and slow release. ${ }^{15,16}$

After IV injection of Tc-99m-sestamibi, initial images are obtained 10-15 minutes after injection which typically show prominent thyroid uptake and a second set of images is obtained at 2-3 hours which showing hyperfunctioning parathyroid gland as a focus of residual activity after much of the thyroid uptake has washed out. ${ }^{17,18}$ SPECT imaging compared with planar imaging has an advantage in delineating the anatomic localization in some cases. However, no study has shown SPECT to be statistically superior in the sensitivity of detection over planar imaging. ${ }^{19,20}$

The most common cause for a false-negative study is small size. Sensitivity for detection of second adenomas or four-gland hyperplasia is lower than for single adenomas. Parathyroid tissue expressing $\mathrm{P}$-glycoprotein (P-gp) and/or multidrug resistancerelated protein (MRP) also fail to accumulate sestamibi. The most common cause for a false-positive parathyroid study is a thyroid follicular adenoma. Interpretation is also difficult in patients taking thyroxine, where thyroid may not be visualized. Other false-positive images have been described with sarcoidosis, thyroid carcinoma, lymphoma and other tumors.

\section{Ultrasound Features}

\section{Typical Parathyroid Nodule}

A typical parathyroid nodule appears as a hemogeneously hypoechoic ovoid to rounded nodule, other shapes noted have been elongated or bilobed parathyroid. On color Doppler, the parathyroid glands show significantly increased vascularity as compared to the adjacent thyroid along with a characteristic arc rim vascularity noted at one pole. The arc rim vascularity is due to the feeding vessel at one of the poles of the parathyroid nodule, usually along the surface adjacent to the thyroid lobe. ${ }^{21-23} \mathrm{~A}$ slight adjustment of the Doppler sensitivity settings may be required to demonstrate the arc and the polar feeding vessel (Figs 1 to 3 ).

\section{Parathyroid Nodule Size}

Parathyroid nodule size may vary from $5 \mathrm{~mm}$ to $45 \mathrm{~mm}$ or more. The largest longitudinal measurement recorded in our series was $45 \mathrm{~mm}$ (Fig. 4). The mean length of the nodule in our series was $18 \mathrm{~mm}$. The measurement of the parathyroid nodules was done in three dimensions and adenoma volume was calculated. These nodule size measurements were compared with histopathology nodule specimen and found to be within $\pm 10 \%$ range in $98 \%$ cases. Sestamibi negative parathyroid nodules in symptomatic hyperparathyroidism were localized on ultrasound in $12 \%$ cases and these measured between $7 \mathrm{~mm}$ and $14 \mathrm{~mm}$. Additional or more than one parathyroid nodule were located on ultrasound in $16 \%$ cases (Figs 5 and 6 ).
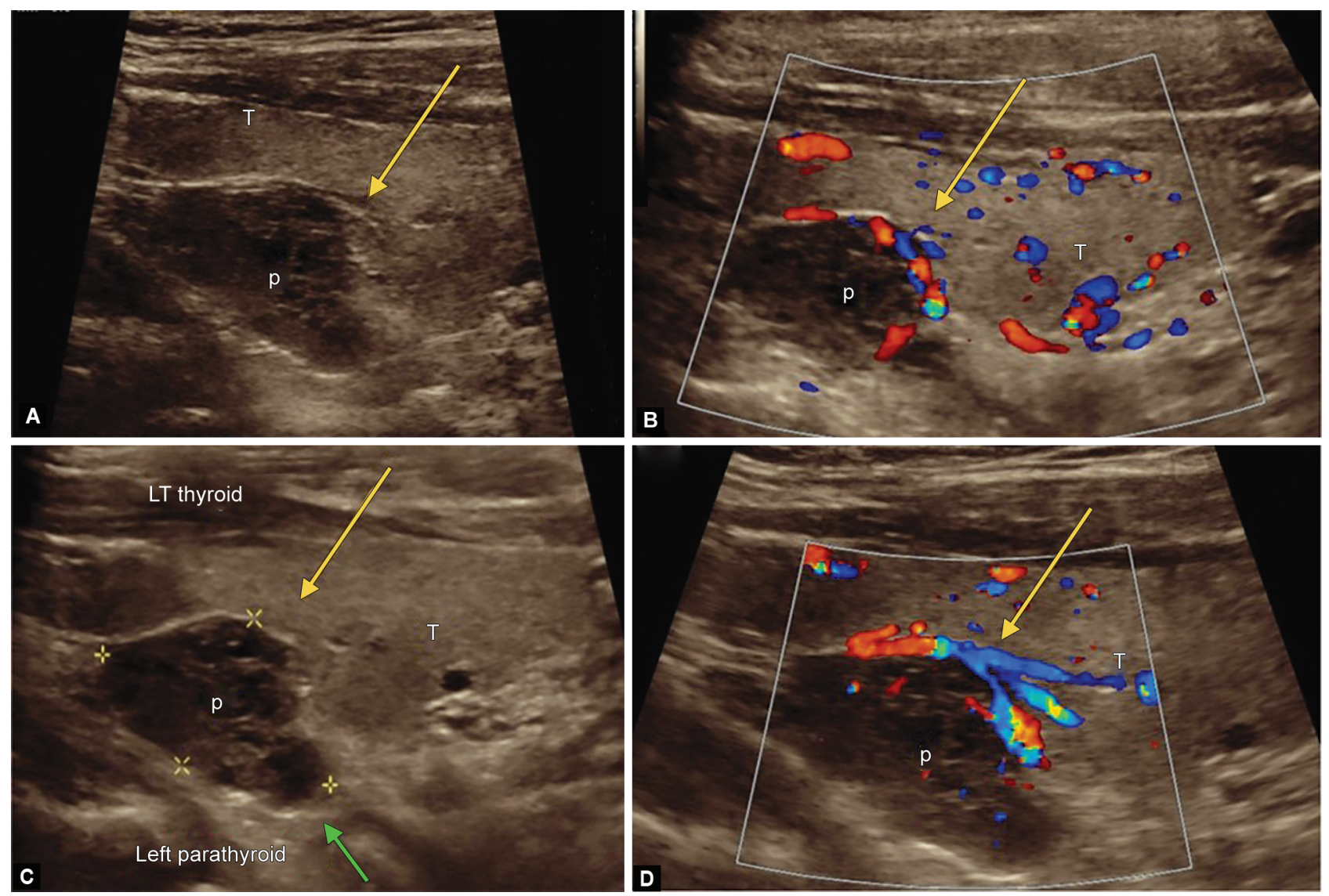

Figs $1 \mathrm{~A}$ to $\mathrm{D}$ : (A and B) Longitudinal ultrasound image of left upper neck in a patient of primary hyperparathyroidism (PHPT) showing a well-defined ovoid hypoechoic nodule located just behind the upper pole of the left lobe of the thyroid. Note the thin echogenic white line (arrow) separating the thyroid from the parathyroid; (C and D) Color Doppler images showing the characteristic arc rim vascularity in the nodule 

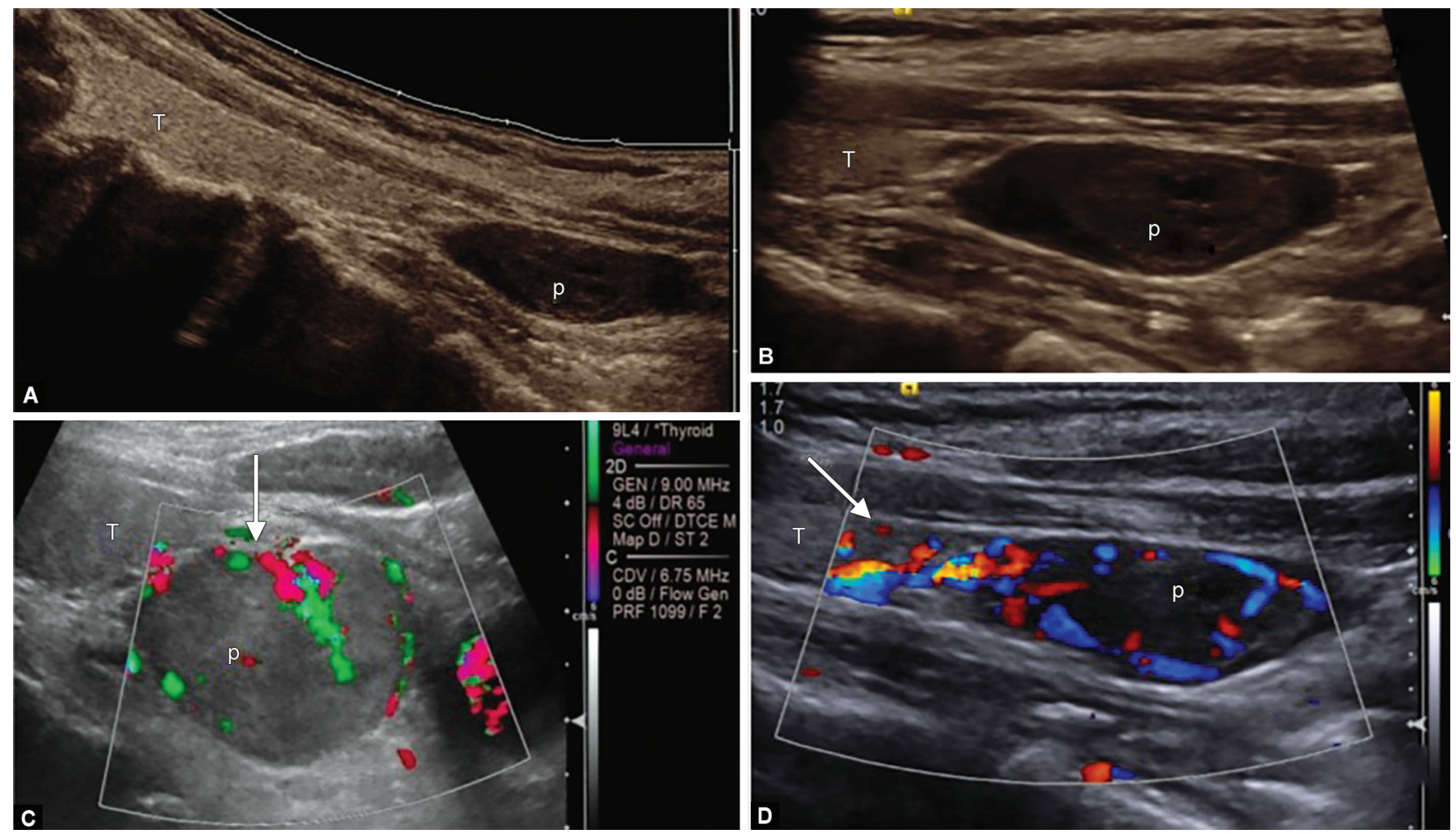

Figs 2A to D: (A and B) Longitudinal extended field of view in a PHPT patient showing thyroid and a typical inferior parathyroid as a well defined ovoid hypoechoic nodule located just below the lower pole of the thyroid; (C and D) Color Doppler images showing the characteristic feeding vessel entering at one of the poles of the parathyroid nodule

\section{A}

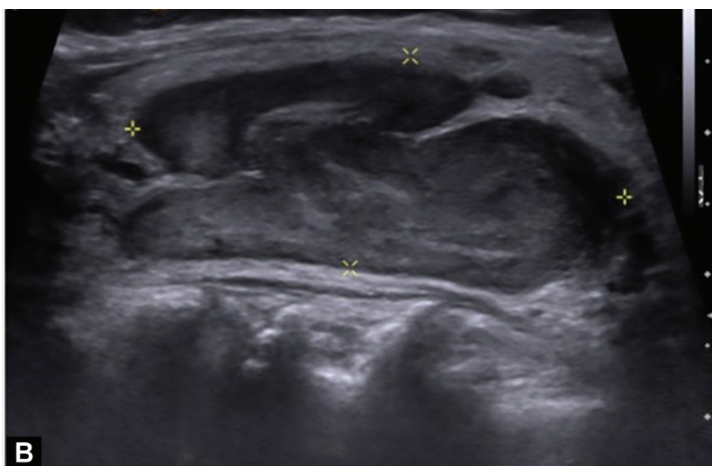

Figs 3A and B: (A) Tc-99m-sestamibi scan for parathyroids in a PHPT patient showing a large right superior parathyroid adenoma as an area of retention of technetium in the right upper neck on the delayed images; (B) Longitudinal ultrasound image showing a large bilobed parathyroid measuring $35 \mathrm{~mm} \times 15 \mathrm{~mm}$, located just behind the upper pole of right thyroid
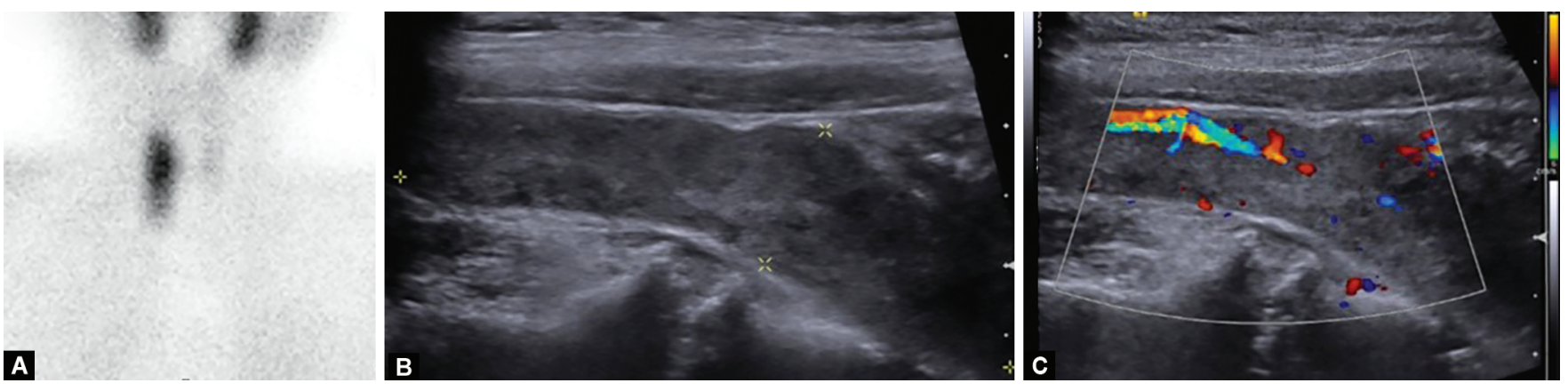

Figs 4A to C: (A) Tc-99m-sestamibi scan for parathyroids in a PHPT patient showing an area of retention of technetium in the right side neck all along with the right lobe thyroid; (B) Longitudinal ultrasound image showing a large elongated parathyroid measuring $45 \mathrm{~mm} \times 15 \mathrm{~mm}$, located behind the whole of right lobe thyroid. The thyroid was small and displaced medially; (C) Color Doppler showing the characteristic arc rim vascularity in the parathyroid nodule 


\section{Atypical Parathyroid Nodules on Ultrasound}

Parathyroid nodules may show cystic changes and calcifications. The cystic changes may be a small cystic area or larger cystic area with small solid soft tissue component in cases of large parathyroid adenomas. Rarely, few thin septations may be seen (Fig. 7). The cystic change is significant from the aspect of the diagnostic evaluation, as on sestamibi only the solid component will show tracer uptake, and the true extent of the size of the lesion can be underestimated (Figs 8 and 9). Ultrasound evaluation is a very valuable adjunct here.

\section{Parathyroid Carcinoma}

Parathyroid carcinoma as a cause of hyperparathyroidism may be suspected clinically when the elevated serum calcium, serum PTH, (often serum calcium is elevated to more than $(>14 \mathrm{mg} / \mathrm{dL}$ ) and serum PTH elevated to more than $(>4,500 \mathrm{pg} / \mathrm{dL}))$ and the clinical symptoms are more severe. It may be associated with genetic syndromes as MEN1. Often when not suspected clinically, ultrasound imaging findings may be the only preoperative clue whether the lesion is likely to be a benign parathyroid adenoma or a parathyroid carcinoma. On ultrasound, parathyroid carcinoma lesion is more heterogeneous and more hypoechoic, it may show heterogeneous vascularity. Its margins with the adjacent thyroid may not be delineated (Fig. 10). It is important to recognize these features as accordingly the preoperative counseling and surgical management changes from minimally invasive parathyroidectomy to an added thyroidectomy or more extensive en bloc surgery.

\section{Parathyroid Cysts}

Parathyroid cysts are rare and usually appear as small clear cysts. They may or may not be functional. Diagnosis is confirmed by histopathology.

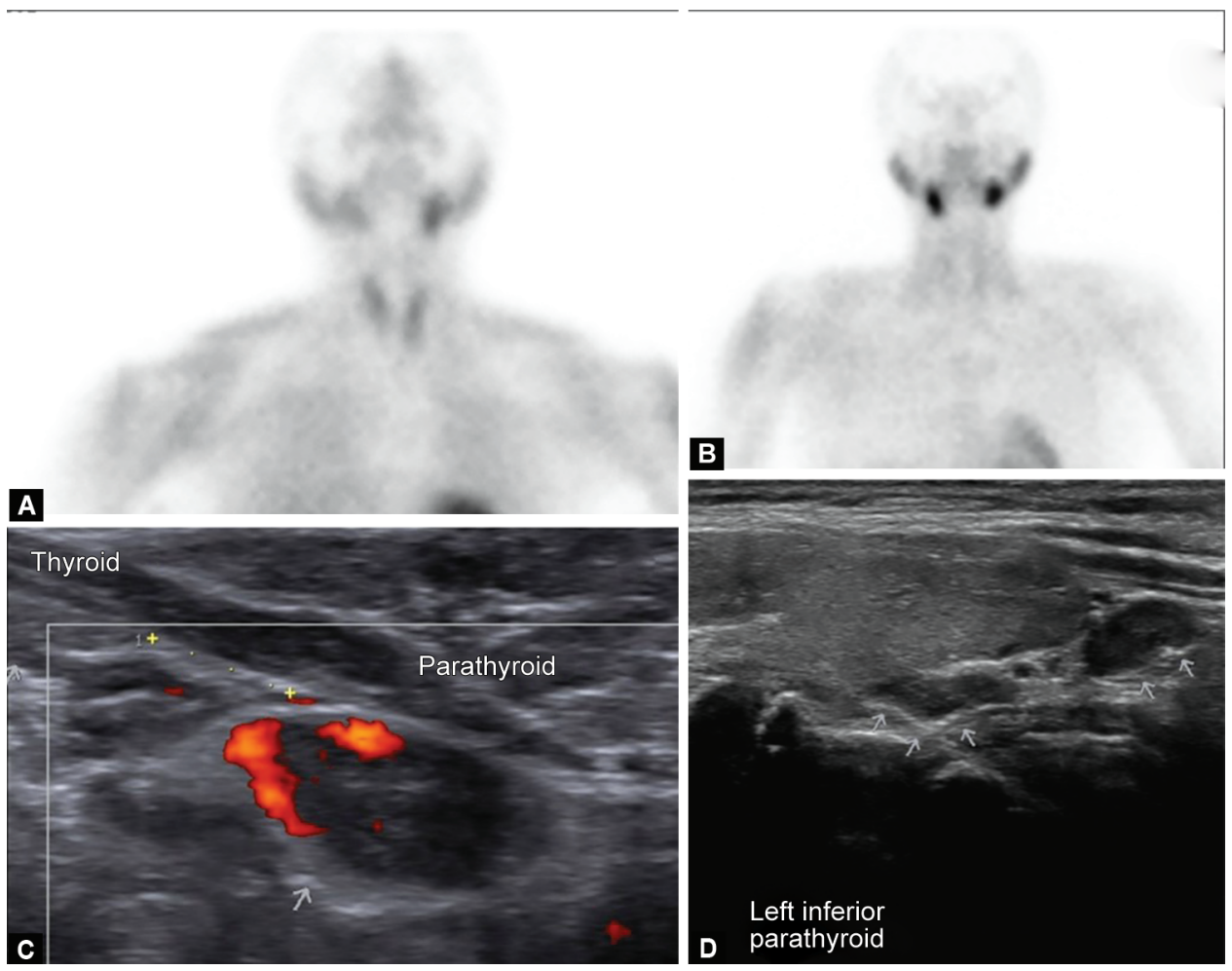

Figs 5A to D: (A and B) Tc-99m-sestamibi scan for parathyroids in a PHPT patient showing no retention of technetium in the neck or mediastinum to suggest any parathyroid adenoma (sestamibi negative hyperparathyroidism); (C and D) Longitudinal ultrasound images of corresponding patients showing typical ovoid parathyroid nodules. One inferior parathyroid nodule is seen in the first patient and two parathyroid nodules are seen in the second patient
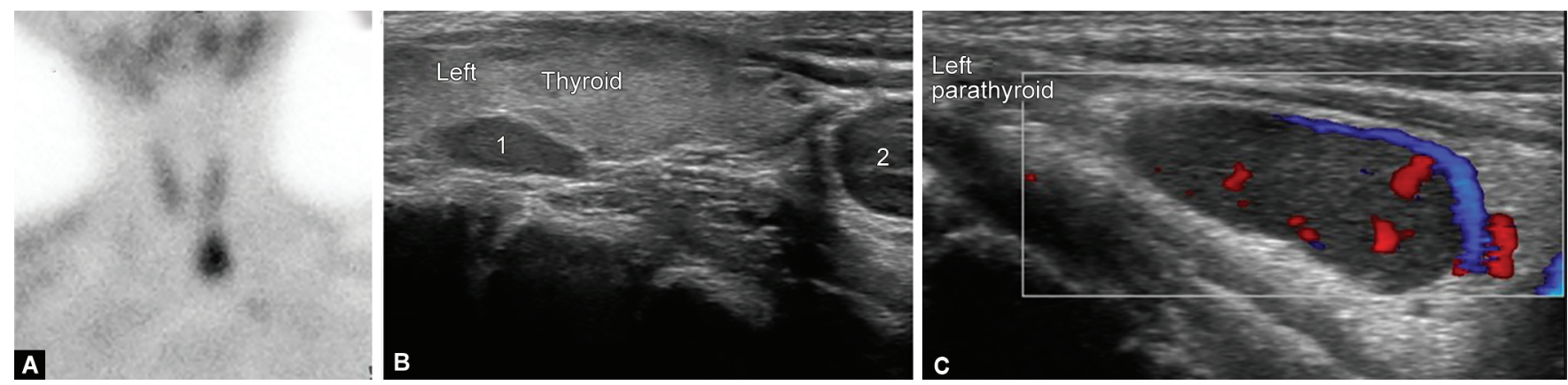

Figs 6A to C: (A) Tc-99m-sestamibi scan for parathyroids in a PHPT patient showing left inferior parathyroid adenoma; (B and C) Longitudinal ultrasound images of the same patient showing an additional small left superior parathyroid nodule (1) along with a large left inferior parathyroid nodule (2) 

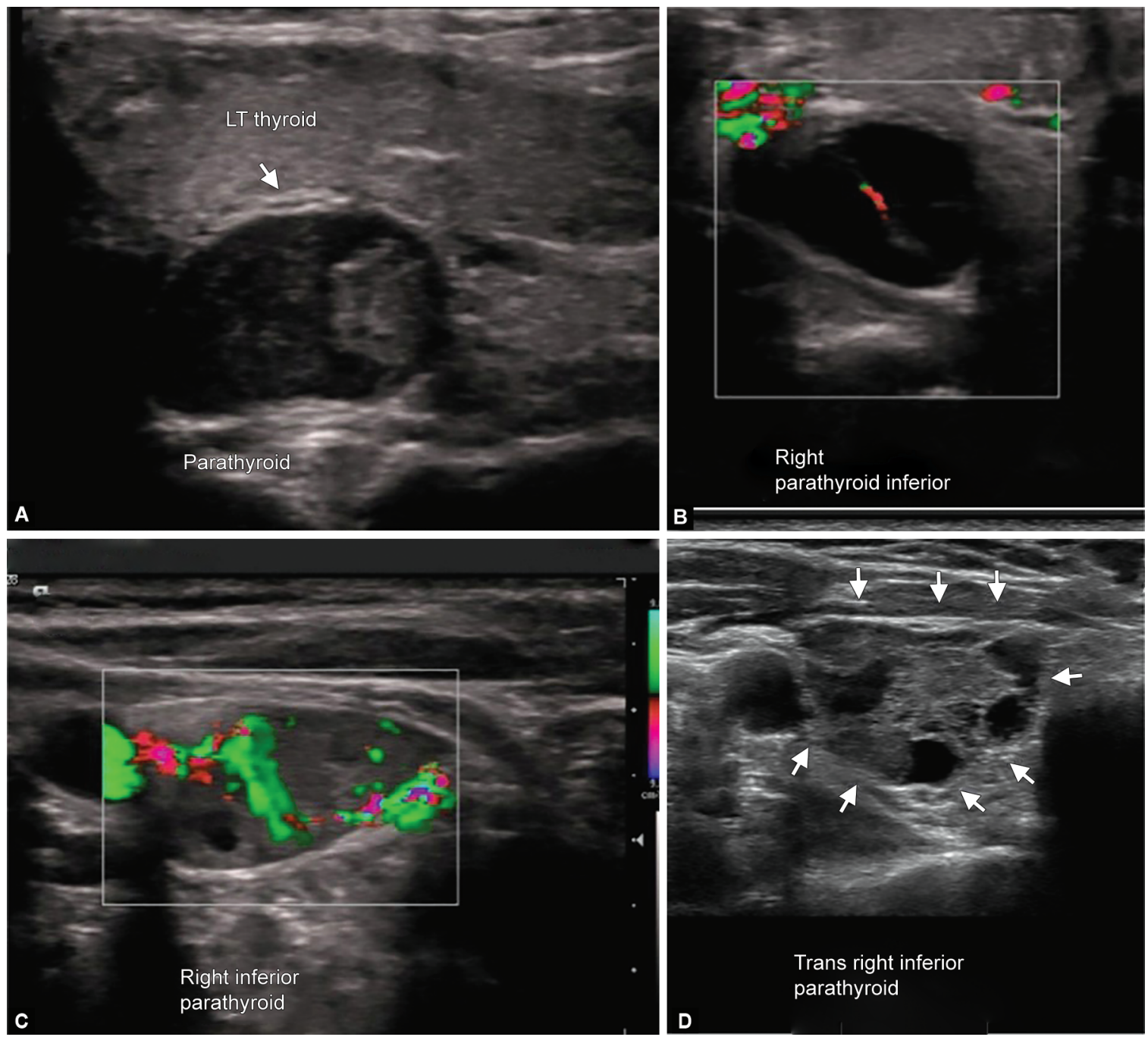

Figs 7A to D: Ultrasound images of atypical parathyroid nodules: (A) Heterogeneous hypoechoic nodule; (B) Parathyroid nodule with enhancing septa; (C) Parathyroid nodule with small cystic change; (D) Parathyroid nodule with multiple cystic changes. Post-surgery histopathology of all the four nodules is parathyroid adenoma
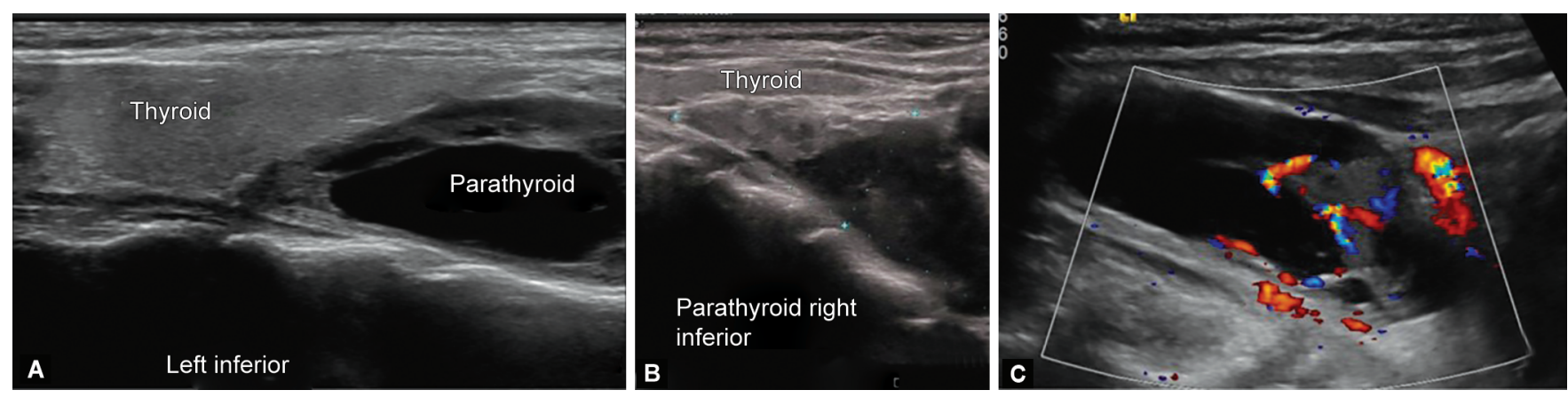

Figs $8 \mathrm{~A}$ to $\mathrm{C}$ : Ultrasound images of parathyroid nodules with large cystic change: (A) Parathyroid nodule with a large cystic change in the central part; (B) Parathyroid nodule with only a small solid soft tissue area in its upper part and a large cystic component dipping deep into the posterior neck; (C) Parathyroid nodule with a large cystic change in the upper part and a small soft tissue component in the lower part. Post-surgery histopathology of all the four nodules was parathyroid adenoma 


\section{Ectopic Parathyroids}

Ectopic parathyroid locations noted were as follows; ${ }^{19,20}$ thyrothymic tract $10.8 \%$, angle of jaw $0.5 \%$, tracheoesophageal and paraesophageal $0.5 \%$, carotid sheath $0.5 \%$, and intrathyroidal parathyroids $2 \%$. Mediastinal ectopic parathyroid was $1.7 \%$. On histopathology, $85.4 \%$ were parathyroid adenoma, $11 \%$ were parathyroid hyperplasia and $3.6 \%$ were parathyroid carcinoma.

\section{Undescended Parathyroid}

The parathyroids originate from the endoderm of the third and fourth pharyngeal pouch, superior from IV, and inferior from the
III, and descend along with the thyroid and thymus to their usual perithyroid locations. An undescended parathyroid ${ }^{4}$ may result as a failure of descent and are usually located at the angle of jaw, just lateral to the submandibular gland (Fig. 11C).

\section{Ectopic along the Thymothymic Tract}

This is the commonest location of the ectopic parathyroids and it usually results in failure of separation of the inferior parathyroids from the thymus during their descent and they may be carried inferiorly beyond the lower pole of the thyroid to up to superior mediastinum. Ectopic inferior parathyroids located up to the angle of manubrium sterni are amenable to visualization by ultrasound.
A

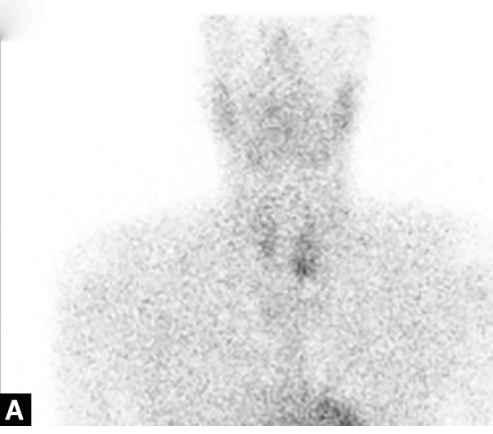

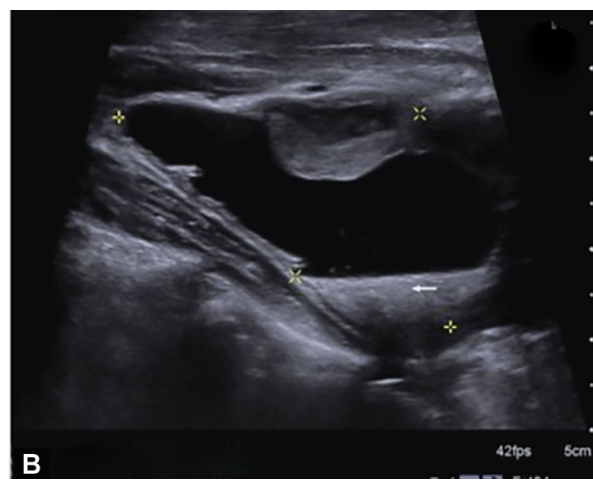

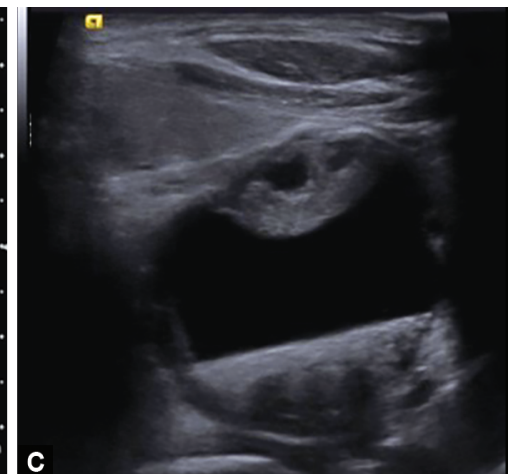

Figs 9A to C: Sestamibi-ultrasound correlation of large cystic parathyroids: (A) Tc-99m-sestamibi scan for parathyroid showing a faint area of tracer retention in the anterior neck corresponding to a possible left inferior parathyroid adenoma; (B and C) Ultrasound images in longitudinal and transverse planes showing a large $43 \mathrm{~mm} \times 22 \mathrm{~mm}$ cystic parathyroid nodule with the layering of internal contents in the dependent part

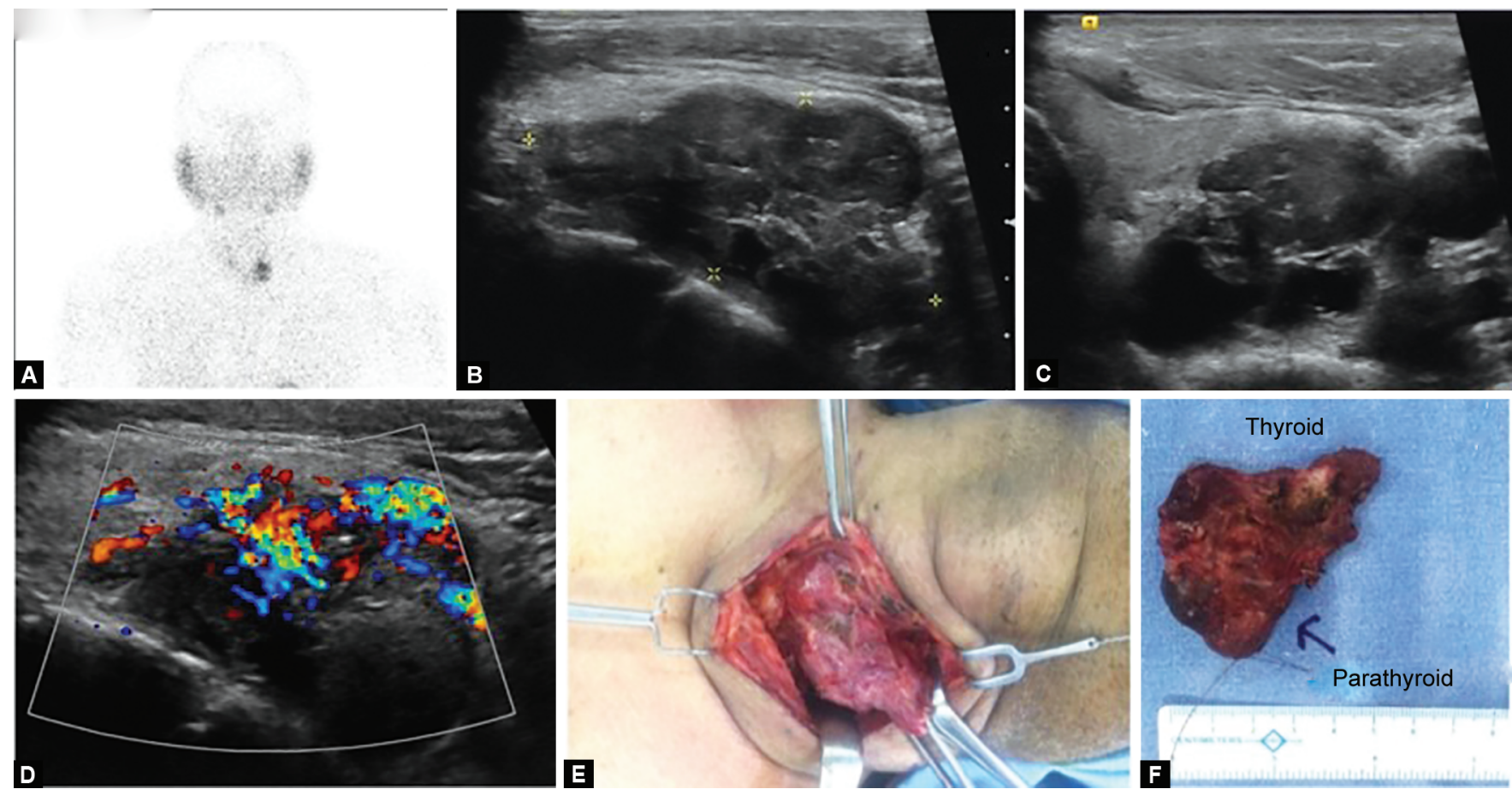

Figs 10 A to F: (A) Tc-99m-sestamibi scan for parathyroid in a patient with PHPT (serum calcium $14 \mathrm{mg} / \mathrm{dL}$ and serum PTH 4,500 pg/mL) showing an area of tracer retention in the anterior neck corresponding to a left inferior parathyroid adenoma; (B to D) Ultrasound images in longitudinal and transverse planes showing a large $39 \mathrm{~mm} \times 22 \mathrm{~mm}$ heterogeneous hypoechoic parathyroid nodule with indistinct margins from the adjacent thyroid in some areas. On color Doppler significant heterogeneous vascularity is noted. Ultrasound findings suggestive of parathyroid carcinoma; (E) At surgery the parathyroid was firm and adherent to the thyroid; (F) Surgical specimen of en bloc thyroid and parathyroid removal. Histopathology confirmed parathyroid carcinoma 

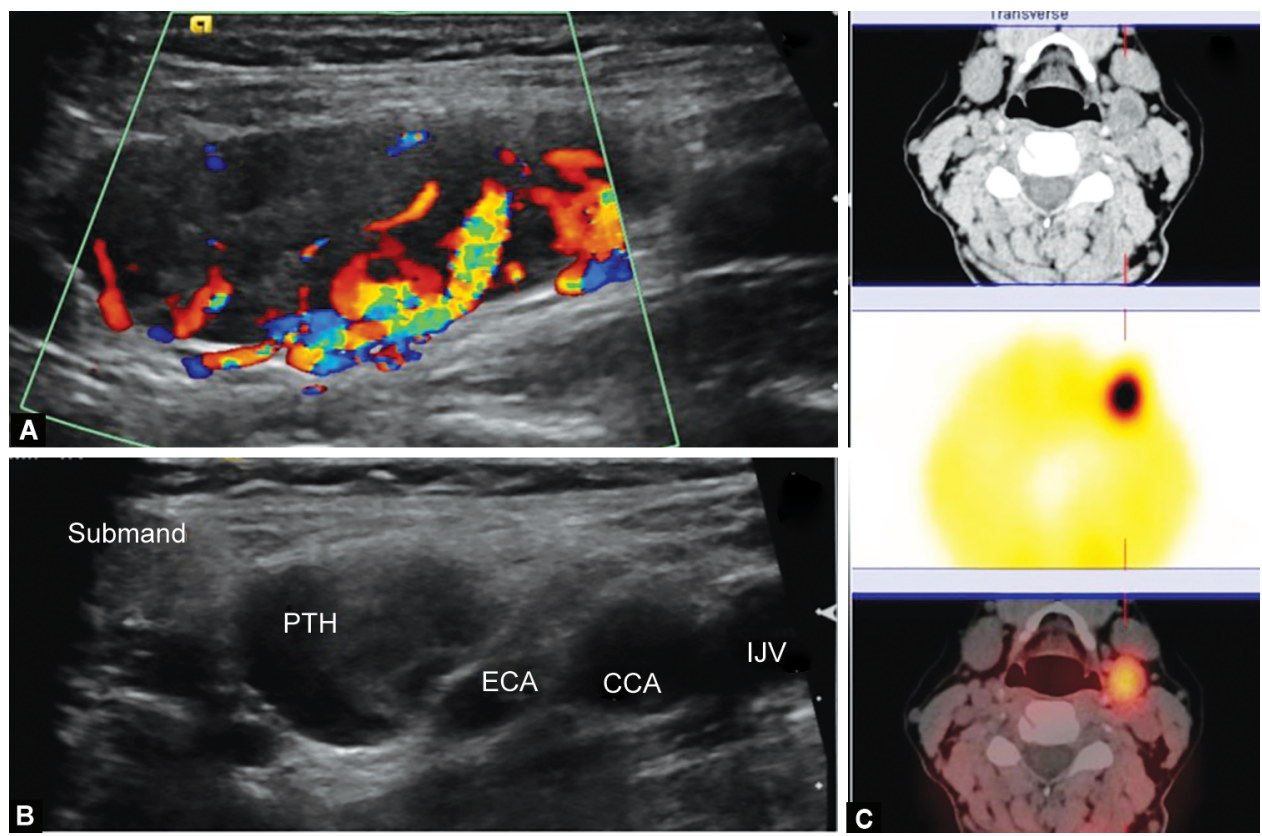

Figs 11A to C: (A and B) A 45-year-old female with PHPT, longitudinal sonogram at the angle of the neck showing a large $30 \times 15 \times 14 \mathrm{~mm}$ well defined ovoid hypoechoic solid nodule with characteristic eccentric arc rim vascularity on color Doppler, located just lateral and adjacent to the left submandibular gland; (C) Sestamibi scan confirmed Tc $99 \mathrm{~m}$ tracer retention at the area on delayed images. Parathyroid adenoma was confirmed at the surgery and subsequent histopathology
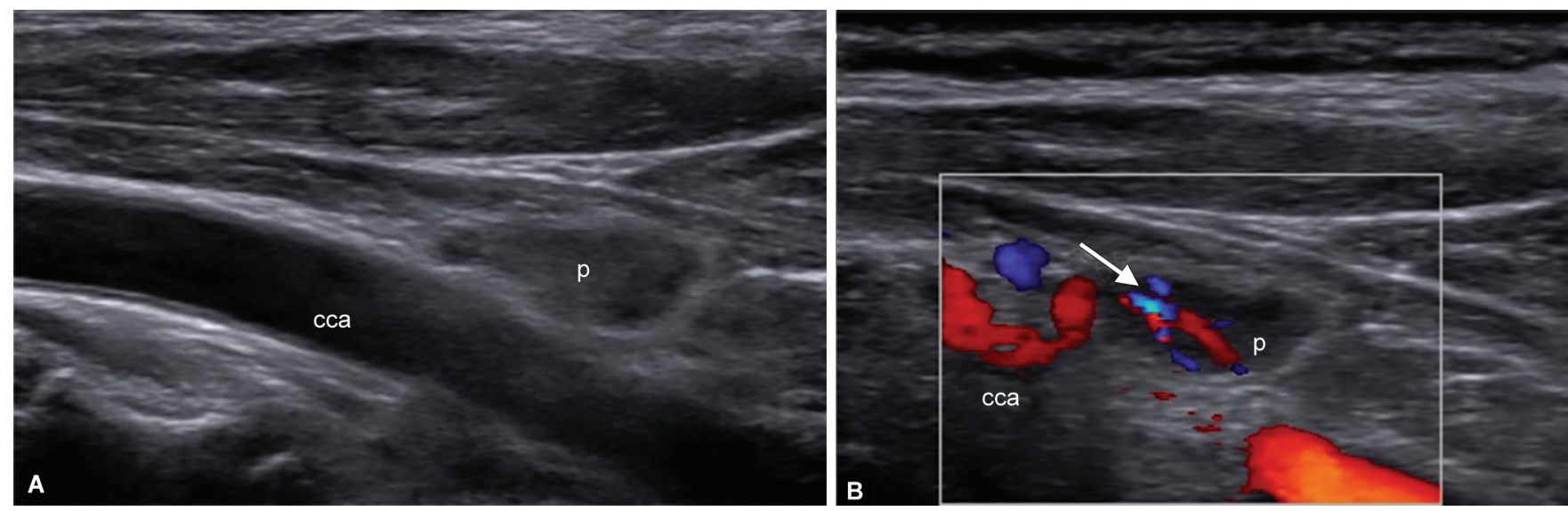

Figs $12 \mathrm{~A}$ and B: Longitudinal oblique sonogram in the left upper lateral neck in a patient with PHPT showing a hemogeneously hypoechoic solid nodule measuring $12 \times 7 \mathrm{~mm}$ located laterally along with the mid-third of the common carotid artery. Note the characteristic arc rim vascularity on color Doppler image

If required a high frequency transducer with a very small footprint such as the hockey stick probe may be utilized to maneuver along the trachea and tracheoesophageal groove.

\section{Ectopic Parathyroid in Carotid Sheath}

Ectopic parathyroids in the carotid sheath are rare, and a thorough transverse and longitudinal scan along the common carotid artery and its bifurcation are required to identify these. Color Doppler settings must be appropriate to elicit the characteristic findings, which could otherwise be masked by the carotid vessel itself (Fig. 12).

\section{Ectopic in Tracheoesophageal/Paraesophageal Groove}

These ectopic parathyroids are located slightly deviated from their path, often laterally and posteriorly are a challenge in diagnosis. The commonest differential diagnosis is from a lymph node in the area of tracheoesophageal or paraesophageal groove. Correlation with another imaging modality, usually Tc-99m-sestamibi or contrast $\mathrm{CT}$ neck is required to establish a definite preoperative diagnosis (Figs 13A and B)

\section{Intrathyroidal Parathyroids}

Intrathyroidal parathyroids are one of the rare locations of ectopic parathyroids and result when the parathyroid glands get trapped in the thyroid during descent.

On MIBI scan there may be a focal area of increased uptake in the thyroid gland, which could be due to parathyroid adenoma or a follicular adenoma. Both follicular adenoma and parathyroid nodules may show increased vascularity and pose a diagnostic challenge in differentiation. When seen, the characteristic arc 

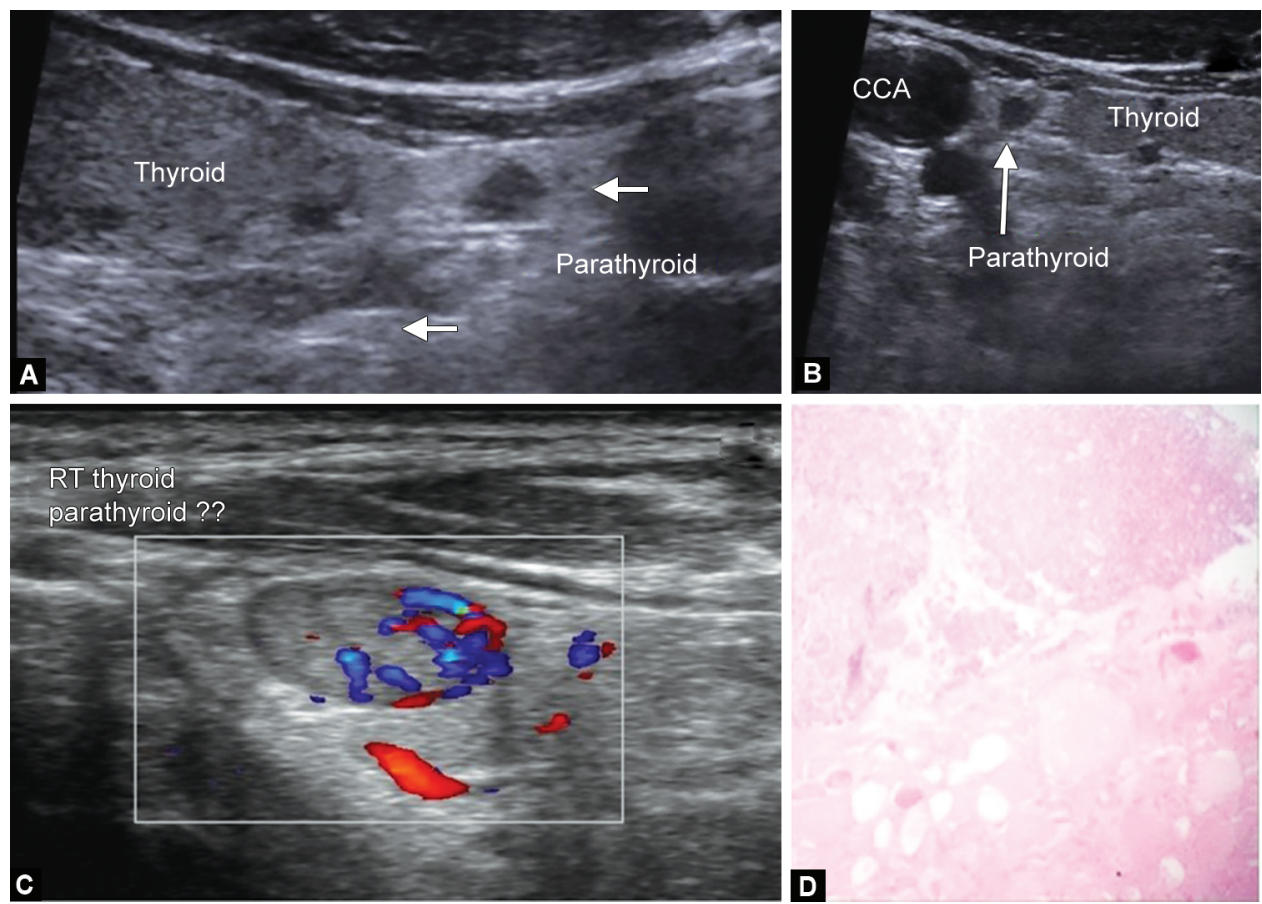

Figs 13A to D: (A amd B) Ultrasound neck in a patient with PHPT showing a hypoechoic solid nodule measuring $11 \times 7 \mathrm{~mm}$ located just below and lateral to the lower tip of left lobe thyroid. The nodule is located anterolateral to the esophagus and is suggestive of a paraesophageal ectopic inferior parathyroid. Findings were confirmed at surgery and histopathology was parathyroid adenoma; (C and D) A hypoechoic nodule in a patient of PHPT with characteristic arc rim vascularity on color Doppler suggestive of the ectopic intrathyroidal parathyroid nodule. Findings were confirmed at surgery and histopathology showed parathyroid embedded in thyroid tissue

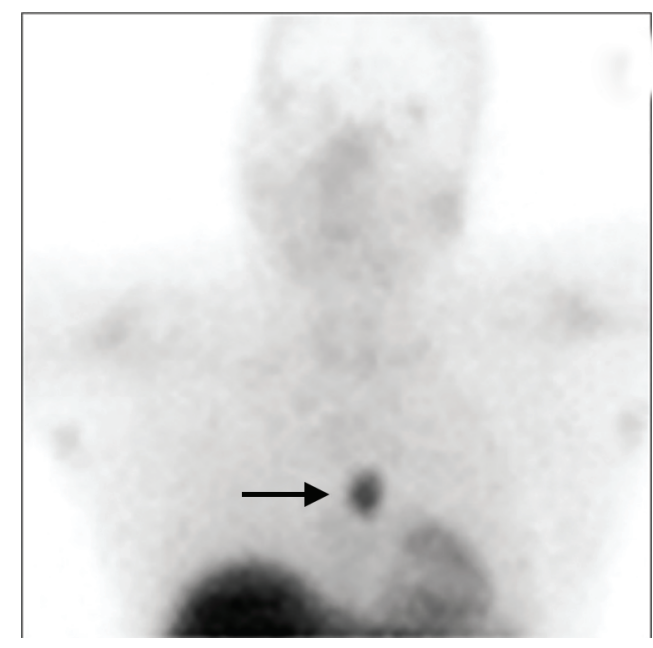

Fig. 14: Tc-99m-sestamibi scan for parathyroids in a PHPT patient showing an area of retention of technetium in the left upper mediastinum suggestive of ectopic left overdescended parathyroid nodule. Surgery and subsequent histopathology confirmed parathyroid adenoma

rim vascularity is highly suggestive of the parathyroid glands. ${ }^{21}$ Another cross-sectional modality such as a dynamic CT Scan may be utilized in difficult cases. True intrathyroidal parathyroids show parathyroid tissue embedded in thyroid tissue on histopathology (Figs $13 C$ and D)

\section{Ectopic Mediastinal Parathyroids}

Parathyroids may be overdescended and reach the mediastinum, 24,25 and by definition are located below the level of clavicles. Ectopic inferior parathyroids move with the thymus and are usually seen in the anterior mediastinum. Ectopic superior parathyroids are usually located in the posterosuperior mediastinum. These may be localized by the sestamibi scan as an area of focal tracer retention in the mediastinum on delayed images (Fig. 14). CT scan is required in these cases for localization.

\section{Conclusion}

Localization of parathyroid adenoma in cases of hyperparathyroidism still remains a diagnostic challenge. Significant anatomical variations and the possibility of supernumerary parathyroids must be remembered. Along with the sestamibi scan, another crosssectional modality must be utilized, either an ultrasound or a CT scan before surgery. An attempt must be made to localize all the four parathyroid glands, over and above the sestamibi findings. An accurate preoperative localization will allow for more focused Parathyroidectomies and reduce the need for BNE and revision surgeries.

\section{References}

1. Lubitz CC, Stephen AE, Hodin RA, et al. Preoperative localization strategies for primary hyperparathyroidism: an economic analysis. Ann Surg Oncol 2012;19(13):4202-4209. DOI: 10.1245/s10434-0122512-2. 
2. Mohebati A, Shaha AR. Anatomy of thyroid and parathyroid glands and neurovascular relations. Clin Anat 2011;25(1):19-31. DOI: 10.1002/ ca. 21220 .

3. Akerström G, Malmaeus J, Bergström R. Surgical anatomy of human parathyroid glands. Surgery 1984;95(1):14-21.

4. Sung JY. Parathyroid ultrasonography: the evolving role of the radiologist. Ultrasonography 2015;34(4):268-274. DOI: 10.14366/ usg.14071.

5. Okabe M, Graham A. The origin of the parathyroid gland. Proc Natl Acad Sci USA 2004;101(51):17716-17719. DOI: 10.1073/ pnas.0406116101.

6. Gilmour JR. The embryology of the parathyroid glands, the thymus and certain associated rudiments. J Pathol Bacteriol 1937;45(5):507522. DOI: $10.1002 /$ path. 1700450304 .

7. Elsheikh E. Superior parathyroid gland approach to the recurrent laryngeal nerve. Head Neck 2017;39(7):1287-1290. DOI: 10.1002/ hed. 24690.

8. Noussios G, Anagnostis P, Natsis K. Ectopic parathyroid glands and their anatomical, clinical, and surgical implications. Exp Clin Endoclinol Diabetes 2012;120(10):604-610. DOI: 10.1055/s-00321327628.

9. Schneider R, Waldmann J, Ramaswamy A, et al. Frequency of ectopic and supernumerary intrathymic parathyroid glands in patients with renal hyperparathyroidism: Analysis of 461 patients undergoing initial parathyroidectomy with bilateral cervical thymectomy. World J Surg 2011;35(6):1260-1265. DOI: 10.1007/s00268-011-1079-6.

10. Uludag M, Yetkin G, Citgez B, et al. The role of cervical thymectomy in surgical treatment of secondary hyperparathyroidism. Bratisl Lek Listy 2011;112(7):385-389.

11. Pattou FN, Pellissier LC, Noël C, et al. Supernumerary parathyroid glands: Frequency and surgical significance in treatment of renal hyperparathyroidism. World J Surg 2000;24(11):1330-1334. DOI: 10.1007/s002680010220.

12. Kaur P, Gattani R, Singhal AA, et al. Impact of preoperative imaging on surgical approach for primary hyperparathyroidism: data from single institution in India. Indian J Endocrinol Metab 2016;20(5):625-630. DOI: 10.4103/2230-8210.190540.

13. Shelley P, Omair RM, Khan Aliya A. Diagnosis and management of primary hyperparathyroidism. BMJ 2012;344:e1013. DOI: 10.1136/ bmj.e1013.
14. Sharretts JM, Kebebew E, Simonds WF. Parathyroid cancer. Semin Oncol 2010;37(6):580-590. DOI: 10.1053/j.seminoncol.2010.10.013.

15. Shane E. Parathyroid carcinoma. J Clin Endocrinol Metabol 2001;86(2):485-493. DOI: 10.1210/jcem.86.2.7207.

16. Desiato $V$, Melis $M$, Amato $B$, et al. Minimally invasive radioguided parathyroid surgery: a literature review. Int J Surg 2016;28(Supp. 1):S84-S93.

17. Eslamy HK, Ziessman HA. Parathyroid scintigraphy in patients with primary hyperparathyroidism: $99 \mathrm{mTc}$ sestamibi SPECT and SPECT/CT. Radiographics 2008;28(5):1461-1476. DOI: 10.1148/rg. 285075055.

18. Wong KK, Fig LM, Gross MD, et al. Parathyroid adenoma localization with 99mTc-sestamibi SPECT/CT: a meta-analysis. Nucl Med Commun 2015;36(4):363-375.

19. Mihai R, Simon D, Hellman P. Imaging for primary hyperparathyroidism - an evidence-based analysis. Langenbecks Arch Surg 2009;394(5):765-784. DOI: 10.1007/s00423-009-0534-4.

20. Roy $M$, Mazeh $H$, Chen $H$, et al. Incidence and localization of ectopic parathyroid adenomas in previously unexplored patients. World J Surg 2013;37(1):102-106. DOI: 10.1007/s00268-0121773-z.

21. Kwon JH, Kim EK, Lee HS, et al. Neck ultrasonography as preoperative localization of primary hyperparathyroidism with an additional role of detecting thyroid malignancy. Eur J Radiol 2013;82(1):e17-e21. DOI: 10.1016/j.ejrad.2012.08.003.

22. Singhal AA, Baijal SS, Sarin D, et al. Ectopic undescended left parathyroid adenoma: diagnosed on ultrasound. J Head Neck Physicians Surg 2017;5(1):44-47. DOI: 10.4103/jhnps. jhnps_6_17.

23. Singhal A, Baijal S, Sarin D. Intrathyroidal parathyroid adenoma in primary hyperparathyroidism: are we overdiagnosing? Case series and learning outcomes. J Head Neck Physicians Surg 2018;6(1):48-53. DOI: 10.4103/jhnps.jhnps_38_17.

24. Hu J, Ngiam K, Parameswaran R. Mediastinal parathyroid adenomas and their surgical implications. Ann R Coll Surg Engl 2015;97(4): 259-261. DOI: 10.1308/003588415X14181254789088.

25. IAS Elhelf, Kademian JC, Moritani T, et al. Ectopic mediastinal parathyroid adenoma localized with four-dimensional CT: a case report. Radiol Case Rep 2017;12(2):247-250. DOI: 10.1016/j. radcr.2017.01.011. 